\title{
The chemical composition of Amazonian plants (*)
}

\section{A Catalogue, edited by Setor de Fitoquímica, INPA, Manaus, Amazonas}

FAMILY

MYRISTICACEAE

OCCurrence: Manaus, Amazonas

TRUNK WOOD :

Sitosterol

2', 4'-Dihydroxy-4,6'-dimethoxydihydrochalcone

Il -2

2',7-Dihydroxy-4, 5'-methylenedioxy-6,8-dimethylflavan

Il -3

2',7-Dihydroxy-4',5'-Methylenedioxy-5,8-dimethylflaven

II -4

1-(2',4'-Dihydroxy-3',5'-dimethylphenyl-3- (2"'-hydrox y-4",5"'-methylenedioxyphenyl)-propane

I) -5

1-(2'-Hydroxy-4'-methoxy-5'-methylphenyl) -3- (2"'-hy droxy-4",5"-methylenedioxyphenyl) -propane II

1-(2',4'-Dihydroxy-3'-methylphenyl)-3- (2"-methoxy-4",5"-methylenedioxyphenyl) -propane

1-(2'-4'-Dihydroxyphenyl) -3-(2"-methoxy-4",5"-methylenedioxyphenyl) -propane

II -8

2,5'-Dihydroxy-7-methoxy-4',5'-methylenedioxy-6,8-dimethylflavane<smiles>COc1ccc(CCC(=O)c2c(O)cc(O)cc2OC)cc1</smiles>

II -2<smiles>[R5]c1cc2c(cc1CCCc1cc([R])c([R])c([R])c1[R6])OCCO2</smiles>

$\begin{array}{llllll} & R_{1} & R_{2} & R_{3} & R_{4} & R_{5} \\ \text { Il - 5 } & \mathrm{Me} & \mathrm{OH} & \mathrm{Me} & \mathrm{OH} & \mathrm{OH} \\ \mathrm{Il}-6 & \mathrm{Me} & \mathrm{OMe} & \mathrm{H} & \mathrm{OH} & \mathrm{OH} \\ \mathrm{Il}-7 & \mathrm{H} & \mathrm{OH} & \mathrm{Me} & \mathrm{OH} & \mathrm{OMe} \\ \mathrm{II}-8 & \mathrm{H} & \mathrm{OH} & \mathrm{H} & \mathrm{OH} & \mathrm{OMe}\end{array}$<smiles>[R6]c1cc2c(cc1C1CCc3c([R])c([R6])c([R6])c([R])c3O1)CCO2</smiles>

$\begin{array}{llllll} & R_{1} & R_{2} & R_{3} & R_{4} & R_{5} \\ \text { Il - 3 } & \mathrm{Me} & \mathrm{OH} & \mathrm{Me} & \mathrm{H} & \mathrm{OH} \\ \text { II }-4 & \mathrm{Me} & \mathrm{OH} & \mathrm{H} & \mathrm{Me} & \mathrm{OH} \\ \mathrm{Il}-9 & \mathrm{Me} & \mathrm{OMe} & \mathrm{Me} & \mathrm{OH} & \mathrm{OH}\end{array}$

REFERENCE :

Silva, Marcelo Sobral (1979) Taken from part of the M. Sc. submitted to Universidade Federal Rural dō Rio de Janeiro.

(*) - Contributions to this catalogue, which will be continued in subsequent issues of this Journal, are invited and should be submitted to address give above. 
FAMILY

LAURACEAE
SPECIE

Aniba lancifolia Kubitzki Rodrigues

OCcurRence: Manaus, Amazonas

TRUNK WOOD :

Sitosterol

2-Hydroxy-4,5-dimethoxy-allylbenzene

(Al - 1)

Lancifolin A- [ $\left(8 \mathrm{R}, 3^{\prime}\right.$ S $)-4$-hydroxy-3,3',4'-trimethoxy-3',6'-dihydro-6'-oxo-8,3'-Neolignan $]$

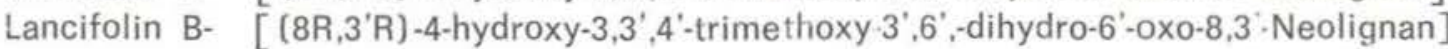

Lancifolin C- [ $\left(8 \mathrm{R}, 3^{\prime} \mathrm{S}\right)-3,4,3^{\prime}, 4^{\prime}$-tetramethoxy-3',6'-dihydro-6'-oxo-8,3'-Neolignan $]$

Lancifolin D- [ (8R, $\left.3^{\prime} R\right)-3,4,3^{\prime}-4^{\prime}$-tetramethoxy 3',6'-dihydro-6'-oxo-8, '-'Neolignan ]

Lancifolin E- [ [8R,3'S) -3,4-methylenedioxy-3',4'-dimethoxy-3',6'-dihydro-6'-oxo-8,3'-Neolignan ]

Lancifolin F- [(8R,3'R)-3,4-methylenedioxy-3',4'-dimethoxy-3',6'-dihydro-6'-oxo-8,3'-Neolignan $]$

$4,5,3^{\prime}, 4^{\prime}$-tetramethoxy-1',2',3',6'-tetrahydro-6'-ox 0-2,0.3'-Neolignan

$(\mathrm{Al}-3)$<smiles>C=CCc1cc(OC)c(OC)cc1O</smiles>

Al -1

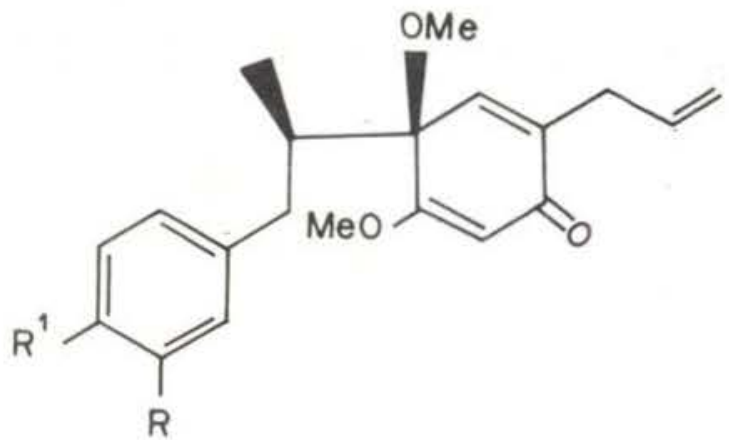

Al - 1

Lancifolin $A \quad R=O M e, R^{1}=\mathrm{OH}$

C $\mathrm{R}=\mathrm{R}^{\mathrm{I}}=\mathrm{OMe}$

E $R, R^{1}=\mathrm{O}_{2} \mathrm{CH}_{2}$<smiles>C=CCc1cc(OC)c(OC)cc1OC1C(OC)=CC(=O)[C@H](CC=C)C1OC</smiles>

AI -3<smiles>[R]c1ccc(C[C@@H](C)[C@]2(OC)C=CC(=O)C(CC=C)=C2)cc1[R]</smiles>

Al - 3

Lancifolin $B \quad \mathrm{R}=\mathrm{OMe}, \mathrm{R}^{\prime}=\mathrm{OH}$

D $\mathrm{R}=\mathrm{R}^{\prime}=\mathrm{OMe}$

F $\mathrm{R}, \mathrm{R}^{\prime}=\mathrm{O}_{2} \mathrm{CH}_{2}$

\section{REFERENCE :}

Diaz Diaz, P.P. (1978) Taken from part of the D.Sc. submitted to Universidade de São Paulo. 